\title{
Lunotriquetral Joint
}

National Cancer Institute

\section{Source}

National Cancer Institute. Lunotriquetral Joint. NCI Thesaurus. Code C139203.

The articulation between the lunate and the triquetral bones in the wrist. 